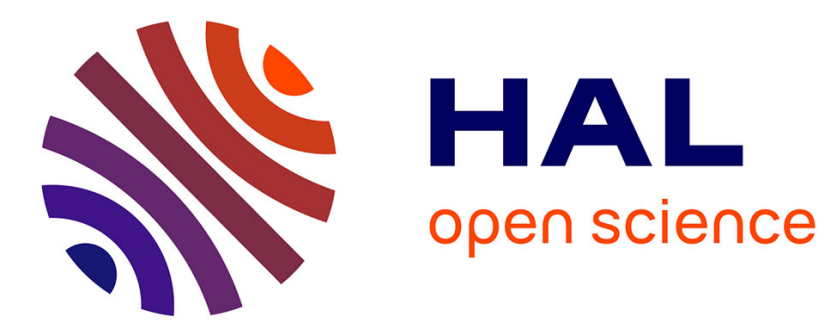

\title{
La forêt et le reboisement dans l'aménagement du territoire rural \\ Jean Gadant
}

\section{To cite this version:}

Jean Gadant. La forêt et le reboisement dans l'aménagement du territoire rural. Revue forestière française, 1987, 39 (S), pp.68-73. 10.4267/2042/25852 . hal-03424636

\section{HAL Id: hal-03424636 \\ https://hal.science/hal-03424636}

Submitted on 10 Nov 2021

HAL is a multi-disciplinary open access archive for the deposit and dissemination of scientific research documents, whether they are published or not. The documents may come from teaching and research institutions in France or abroad, or from public or private research centers.
L'archive ouverte pluridisciplinaire HAL, est destinée au dépôt et à la diffusion de documents scientifiques de niveau recherche, publiés ou non, émanant des établissements d'enseignement et de recherche français ou étrangers, des laboratoires publics ou privés. 


\section{LA FORÊT ET LE REBOISEMENT DANS L'AMÉNAGEMENT DU TERRITOIRE RURAL}

J. GADANT

On a dit - un peu vite - de la forêt qu'elle est "une production hors du temps et une économie hors de l'économie". Il est vrai que la longueur des délais qui sépare la plantation de la coupe, l'absence de récoltes annuelles et régulières, une médiocre rentabilité financière, donnent à ce secteur économique de la forêt une originalité certaine.

\section{LA COMPLÉMENTARITÉ DE L'AGRICULTURE ET DE LA FORÊT}

La moitié des exploitations agricoles ont des parcelles boisées, dont la superficie totale approche trois millions d'hectares; ainsi cette forêt dite "paysanne " représente le tiers des forêts privées, le double des forêts domaniales, l'équivalent du patrimoine forestier communal. Mais dans les zones de moyenne montagne, le pourcentage des exploitations agricoles qui possèdent des forêts est beaucoup plus élevé : plus de $80 \%$ en Corrèze, en Aveyron, en Dordogne, etc.

Cette forêt paysanne souvent mal valorisée recèle des possibilités de productivité et de progrès. Ce potentiel de 500000 exploitants agricoles apparaît particulièrement apte à la valoriser par leur présence, la connaissance qu'ils ont du terroir, leur savoir-faire paysan, des matériels utilisables en forêt et le temps dont ils peuvent disposer en morte saison agricole. La sylviculture et l'exploitation forestière peuvent apporter à un agriculteur des zones défavorisées, un complément appréciable de revenu: coupes,

- soit qu'il possède quelques hectares boisés où il puisse réaliser périodiquement des

- soit qu'il effectue des prestations de services (surveillance, gestion, travaux) dans des forêts voisines, appartenant à d'autres.

Ce développement forestier, à partir du potentiel agricole, peut contribuer à créer de l'activité en zones défavorisées et à viabiliser des exploitations agricoles par l'apport d'un revenu forestier complémentaire. Des pays comme la Finlande et l'Autriche, où une forte proportion de la forêt privée est intégrée dans des exploitations agricoles, ont su bâtir une politique forestière particulièrement dynamique à partir de leur potentiel agricole.

Par ailleurs, la forêt a souvent eu bénéfice à s'inspirer des méthodes utilisées en agriculture et à se rapprocher de ses structures; ainsi, le transfert de l'enseignement forestier à l'enseignement agricole, et le transfert de la recherche forestière à I'Institut national de la Recherche agronomique lui ont été particulièrement bénéfiques. Au cours des trente dernières années, les techniques de reboisement se sont constamment perfectionnées en faisant appel aux techniques agronomiques: le travail du sol, la fertilisation, la sélection. Aujourd'hui, la populiculture, la 


\section{Le reboisement}

ligniculture, la culture de taillis à courte révolution sont des méthodes purement agricoles et de véritables cultures d'arbres.

En matière de développement de la production forestière et de promotion du reboisement, il y a des cousinages agro-forestiers très nombreux:

- Les CETEF (Centre d'étude technique forestière) et les GVF (Groupement de vulgarisation forestière) sont inspirés des CETA et des GVA de la vulgarisation agricole.

- L'IDF (Institut pour le développement forestier) appartient à la famille des instituts techniques agricoles spécialisés.

- Le groupement forestier est frère du GFA (Groupement foncier agricole) : mais cette fois c'est le premier qui a inspiré le second.

- Il en est de même du groupement d'exploitation forestière en commun et du GAEC.

- Le plan de gestion forestière et le plan de développement agricole poursuivent un même but, permettant l'un et l'autre de planifier des opérations de mise en valeur.

- Les associations syndicales de gestion forestière de 1985 sont inspirées de la législation sur les associations foncières pastorales de 1972.

\section{LA FORẼT DANS L'AMÉNAGEMENT RURAL}

Le patrimoine forestier français est le plus étendu d'Europe occidentale; il couvre près de la moitié de la superficie boisée des dix pays de la Communauté économique européenne. Par ailleurs, l'effort de reboisement mené depuis trente ans va mettre la France en mesure de mieux satisfaire ses besoins en bois. Mais cette vision globale sur l'importance du patrimoine forestier ne rend pas compte de réalités régionales beaucoup plus nuancées. En particulier, il est très inégalement réparti sur le territoire rural; les Landais d'Aquitaine engloutis dans leur immense pineraie peuvent aspirer à la clairière; par contre, les habitants des grandes plaines du Nord manquent d'espaces boisés pour y rompre la monotonie céréalière.

En moyenne montagne, il est arrivé que la progression de la forêt sur des terres abandonnées par l'agriculture ait été abusivement envahissante; après que des subventions aient incité à la plantation, il a donc fallu imaginer une procédure contraignante de limitation du droit de reboisement pour contrôler son avancée. Par contre, dans l'environnement des grandes villes et des pôles touristiques, l'urbanisation a fait reculer le taux de boisement de façon parfois excessive, et il a fallu réglementer le défrichement pour s'opposer à une inquiétante régression de la forêt.

Bien qu'elle soit implantée dans le long terme et que ses évolutions soient très lentes, la forêt n'a pas ce caractère immuable et statique que certains imaginent. Ses limites bougent. Trop importante ici, elle est insuffisante ailleurs. C'est pourquoi, afin de maîtriser ces évolutions, une politique forestière doit avoir deux volets: I'un offensif d'expansion par le reboisement de terres abandonnées, l'autre défensif de protection et de conservation des forêts existantes.

La forêt constitue donc un élément de l'aménagement du territoire, dont la présence suffisante, sans être excessive, doit être répartie au mieux sur le territoire et planifiée dans les documents d'aménagement de l'espace. Bien entendu, on voit mal comment ces politiques de développement et de protection pourraient être mises en œuvre sans se préoccuper aussi de tout l'espace qui environne la forêt et sans agir globalement sur l'ensemble rural.

La panoplie des moyens réglementaires est bien pourvue pour assurer une place convenable de la forêt sur le territoire rural: réglementation du reboisement, plan d'occupation des sols, maîtrise des coupes, contrôle des défrichements, etc. 


\section{J. GADANT}

La loi de décentralisation en donnant du pouvoir aux collectivités territoriales et aux élus locaux met à leur disposition de nouvelles procédures de développement local qui ouvrent à la forêt de nouvelles perspectives.

\section{LA FORÊT DANS LE DÉVELOPPEMENT LOCAL}

On parle de la forêt française et du problème forestier au singulier, dans la vision simpliste et unitaire d'un secteur qui serait bien défini et parfaitement homogène. Certains dénoncent le morcellement de la forêt, sa mauvaise gestion, sa sous-exploitation; d'autres traitent ces maux de faux problèmes.

En vérité, les uns et les autres ont à la fois tort et raison; sur ce thème de la forêt, tout peut être dit et son contraire. En effet, l'expression "la forêt française " et les appréciations globales la concernant travestissent une réalité fort complexe et toujours nuancée. Ce qui est vrai ici apparaît erroné ailleurs. II est donc déformant de traiter de la forêt et du bois au singulier et depuis des bureaux parisiens. Néanmoins, la politique forestière n'a pas été décentralisée et continue de relever des pouvoirs de l'État. Mais la loi forestière de 1985 module localement son application et la cadre au moyen d'orientations régionales de production.

Cependant, s'il est déformant d'évoquer la forêt au niveau national, il en est de même au niveau d'une région, où les disparités demeurent très grandes et où tous les types de forêts sont aussi représentés. C'est pourquoi, les politiques régionales de valorisation et de protection de la forêt devront aussi se décentraliser largement sur les petites régions où les vrais problèmes peuvent être clairement posés et efficacement traités. Cela implique de responsabiliser davantage les collectivités locales et de mener des actions dans le cadre des procédures décentralisées de développement local. Les chartes intercommunales d'aménagement et de développement ouvrent aux forestiers d'intéressantes perspectives pour un bon traitement des problèmes de la forêt et des industries du bois.

Ce traitement de la forêt dans une conception plus décentralisée et plus globale de l'amenagement et du développement permettra aux forestiers de rompre avec deux types d'isolement dans lesquels ils ont été trop enfermés dans le passé:

- isolement de l'aval industriel qui conditionne la prospérité de la forêt,

- isolement de l'aménagement du territoire rural dans lequel la forêt se trouve enracinée. La politique forestière doit nécessairement entretenir des liaisons et des complémentarités avec la politique agricole.

\section{LA FORÊT DANS L'AMÉNAGEMENT FONCIER}

Les zones de moyenne montagne connaissent le déclin économique le plus accentué malgré des potentialités naturelles mal valorisées d'herbages et de forêts. Tous ces territoires sont affectés des mêmes handicaps : dépeuplement, vieillissement de la population, isolement, sols inaptes à l'intensification, extension désordonnée de la forêt, imbrication confuse des activités agricoles et forestières, morcellement foncier. Leur valorisation implique un effort d'aménagement et d'organisation foncière.

\section{La réglementation du boisement}

L'intensification de la production agricole et l'exode rural ont libéré des terres agricoles qui ont été reboisées. Mais un développement mal maîtrisé de ces plantations, au plan foncier, a suscité 
parfois l'hostilité des agriculteurs locaux, leur colère allant jusqu'à l'arrachage des jeunes plantations. Le reboisement ètait ressenti par eux comme une invasion appauvrissante, d'autant plus qu'il était réalisé au protit d'ètrangers au pays.

C'est pourquoi l'article 52-1 du Code rural, issu de la loi d'orientation agricole de 1960, est venu donner le moyen juridique de s'opposer à ce désordre. La procédure consiste à cartographier la zone agricole dans laquelle tous semis ou plantations d'essences forestières seront interdits pour quatre ans, la zone forestière où le boisement pourra se développer librement, éventuellement une zone intermédiaire réglementée dans laquelle les plantations seront soumises à des contraintes d'éloignement des fonds voisins ou à des autorisations administratives préalables.

Bien entendu, cette réglementation, totalement négative pour la forêt, entraîne une limitation du droit du propriétaire, empêché de disposer librement de sa terre ou bien contraint de la vendre ou de la louer, parfois à vil prix. Par ailleurs, elle n'apporte pas de vraie solution, puisque la parcelle frappée d'interdits s'embroussaillera et se boisera naturellement si elle n'est pas effectivement cultivée. C'est pourquoi cette réglementation ne constitue qu'une mesure provisoire et défensive; elle n'a d'intérêt que dans la mesure où elle prépare une action d'ensemble de réorganisation toncière.

Le district pilote de reboisement de 1962 et le périmètre d'action forestière de 1971 ouvraient une première voie. Mais ils ont montré aux torestiers qu'il était totalement inefficace de concevoir une politique cohérente de reboisement en isolant celle-ci de préoccupations plus globales d'aménagement et de développement rural. II fallait engager une procédure d'aménagement foncier, à la fois agricole et forestier.

\section{Un aménagement foncier global}

La société pour la Mise en valeur de l'Auvergne et du Limousin (SOMIVAL.) créée en 1962, avait entrepris de développer les trois potentialités du Massif Central: I'agriculture, le tourisme et la forêt. Sa division forestière m'avait été confiée. Des conditions écologiques favorables à une production ligneuse de haut rendement, l'importance des friches dans le paysage rendaient l'entreprise enthousiasmante et, a priori, théoriquement facile. Mais rapidement il fallut déchanter : un morcellement foncier considérable rendait la mobilisation des terres incultes particulièrement ditticile pour organiser ce reboisement rationnel que la Société de mise en valeur voulait promouvoir. Une étude qu'elle réalisa en 1963, intitulèe "le reboisement et les structures foncières", analysait le problème et proposait une stratégie.

D'abord, elle désignait le coupable: "l'anarchie foncière sur un territoire agricole accidenté, où le repli démographique est désordonné, et que les systèmes de polyculture et les héritages du passé ont morcelé à l'extrême".

Ensuite elle prescrivait le remède: "un aménagement foncier global du territoire communal, traitant simultanément l'affectation agricole, l'affectation forestière étroitement imbriquée et dépendante et le regroupement du bâti ".

Une méthode d'aménagement foncier globale était proposée. Elle substituait une procédure coordonnée d'aménagement foncier agricole et forestier à toutes les procédures sectorielles existantes qui s'ignorent, parfois se contrarient, et dont la mise en cuvre isolée est inefficace. La méthode d'aménagement proposée mariait dans une seule procédure toutes les dispositions foncières éparpillées dans les codes rural et forestier. L'idée était simple: ne plus faire d'aménagement agricole sans penser aussi à la forêt; ne plus faire d'aménagement forestier sans se préoccuper des problèmes agricoles.

Et pourtant les moyens de rénovation de ces structures foncières en désordre ne manquaient pas; les codes rural et forestier recélaient un arsenal bien fourni de procédures. Mais le 
remembrement excluait la forêt de ses périmètres. Les SAFER limitaient leurs interventions au seul secteur agricole. Par ailleurs, la manipulation de tous ces outils d'aménagement, indépendamment les uns des autres, à l'initiative d'administrations différentes conduisait à l'inefficacité. A un problème foncier très global, touchant l'agriculture, la forêt et les sols construits, il fallait apporter une solution plus compacte et plus cohérente.

Les lois foncière et forestière de 1985 l'ont apportée en dotant la Commission communale d'aménagement foncier d'une panoplie de bons moyens pour lui permettre de promouvoir un amenagement foncier plus global du territoire rural, sans jamais exclure la forêt :

- les divers modes de remembrement: remembrement agricole et rural, remembrement simplifié, remembrement-aménagement, remembrement forestier, remembrement agricole et forestier ;

- les échanges de droits propriété et d'exploitation, qu'ils soient agricoles ou forestiers ;

- la mise en valeur des terres incultes par l'agriculture et le reboisement;

- la réglementation du boisement ;

- la nouvelle procédure de réorganisation foncière.

Les dispositions du Code rural applicables à ces différents modes d'aménagement foncier sont désormais regroupées dans un "tronc commun " et s'intègrent dans une même procédure : mise en place de la commission communale, choix des modes d'aménagement foncier, délimitation des périmètres, avis de la commission départementale, etc...

Ainsi, désormais, la commission communale dispose d'une véritable "boîte à outils de l'aménagement rural » pour traiter à la fois les zones agricoles, forestières et bâties.

\section{L'aménagement foncier agricole et forestier}

L'aménagement est réalisé dans le cadre communal, éventuellement intercommunal, dans les mêmes conditions qu'une opération de remembrement classique. En particulier, il est conduit par une commission communale d'aménagement foncier dont les décisions peuvent être réformées par une commission départementale. Cet aménagement foncier agricole et forestier poursuit deux objectifs:

- d'une part, il localise les diverses natures de culture sur les terroirs les mieux appropriés et les concentre sur des zones homogènes. Ainsi on ne cultivera plus au milieu de la forêt et on ne boisera plus parmi les terrains agricoles;

- d'autre part, il regroupe les propriétés et les exploitations à l'intérieur d'affectations culturales définies. En effet, il conviendra de mettre un terme au bricolage sur des exploitations agricoles de petite taille et sur des propriétés forestières minuscules ou éclatées entre plusieurs parcelles.

La localisation des propriétés agricoles et forestières sur les zones à affecter à l'agriculture et sur celles à affecter à la forêt nécessite des échanges parcellaires en fonction des intentions des propriétaires et des exigences de l'intérêt général. La loi a donc créé une procédure de remembrement agricole et forestier. Son originalité est de permettre des échanges entre parcelles non boisées et parcelles boisées, afin de placer l'agriculteur dans l'espace agricole, le sylviculteur dans l'espace forestier et déboucher ainsi sur un véritable zonage d'activités.

Mais on trouvera des propriétaires inintéressés et qui préfèrent vendre. II fallait donc prévoir aussi l'intervention d'un opérateur foncier qui puisse acheter, revendre, apporter à des structures de mise en valeur collective. La loi fait intervenir les SAFER.

Parallèlement, la commission communale d'aménagement foncier agricole et forestier dresse les inventaires des terres incultes, des biens vacants et sans maître, des propriètés sectionales 
abandonnées et met en œuvre les procédures de récuperation et de mise en valeur les concernant. Elle pourra aussi utilement procéder à une délimitation des espaces écologiques ou paysagers devant faire l'objet d'une protection particulière.

De même que dans un remembrement agricole classique, tout ce réaménagement parcellaire agricole et forestier est prolongé par des travaux d'équipement indispensables à la mise en valeur. Ils sont programmés par la commission communale et pris en charge par une association foncière qui pourrait également être chargée des travaux de boisement si les propriètaires le desirent.

En répartissant le territoire en zones agricoles cultivées par des agriculteurs et en zones boisées ou à boiser gérées par des forestiers, l'action d'aménagement met un terme au conflit qui opposait l'agriculture et la forêt. Ainsi, le vieux concept d'équilibre agro-sylvo-pastoral pourrait enfin prendre du sens.

La crèation du Corps des ingénieurs du Génie rural, des Eaux et des Forêts, ainsi que l'institution des Directions départementales de l'Agriculture, en 1965, avaient consacré I'heureux rapprochement du rural, de l'agricole et du forestier. La nouvelle organisation apportait aux forestiers de larges ouvertures et leur offrait la possibilité d'un traitement plus global des problèmes de la forêt.

Vingt ans après, à l'occasion de la réforme des services extérieurs de 1985 , le $F$ forestier ajouté à la DRA (Direction régionale de l'Agriculture) et à la DDA (Direction départementale de l'Agriculture), pour en faire des DRAF et des DDAF, est venu à nouveau souligner l'importance du rapprochement de l'agricole et du forestier dans la vie administrative.

La rècente création d'une Direction de l'Espace rural et de la Forêt est un nouveau pas vers un aménagement plus global et une mise en valeur mieux coordonnée du territoire rural.

\section{J. GADANT}

Ingènieur génèral du G.R.E.F.

Prèsident de la 4ème Section Forêts-Bois

CONSEIL GENERAL DU GÉNIE RURAL,

DES EAUX ET OES FORETTS

30, rue Las Cases

75340 PARIS CEDEX 07 